\title{
3 Research Square \\ The Mutational Landscape of Upper Gastrointestinal Adenocarcinomas- A Study of Similarities and Differences
}

Kritika Krishnamurthy ( $\sim$ kritikakrishnamurthy@yahoo.com )

Mount Sinai Medical Center https://orcid.org/0000-0003-0772-0150

Sophia Navajas Urioste

Florida International University Herbert Wertheim College of Medicine

Mike Cusnir

Mount Sinai Medical Center

Michael Schwartz

Mount Sinai Medical Center

Sarah Alghamdi

Mount Sinai Medical Center

Vathany Sriganeshan

Mount Sinai Medical Center

Robert Poppiti

Mount Sinai Medical Center

\section{Research Article}

Keywords: Upper gastrointestinal adenocarcinomas, genetics, next generation sequencing, APC, PIK3, PIK3 class 1.

Posted Date: August 26th, 2021

DOl: https://doi.org/10.21203/rs.3.rs-827155/v1

License: (a) (1) This work is licensed under a Creative Commons Attribution 4.0 International License. Read Full License

Version of Record: A version of this preprint was published at Pathology - Research and Practice on February 1st, 2022. See the published version at https://doi.org/10.1016/j.prp.2022.153830. 


\section{Abstract}

\section{Purpose-}

The gastrointestinal tract is home to a wide variety of neoplasms. Gastrointestinal adenocarcinomas display distinct prognostic patterns. With the advent of next generation sequencing, attempts are being made to delineate distinct molecular characteristics of these adenocarcinomas from adjoining anatomical sites.

Methods-

Thirty-seven cases of upper gastrointestinal adenocarcinomas including those of the esophagus, gastroesophageal junction, stomach, small intestine and gallbladder were retrieved. Next generation sequencing data consisting of base substitutions, copy number variations, indels and rearrangements, in 324 genes, were analyzed for recurrent genetic abnormalities. Statistical analysis was performed using IBM SPSS25 and SAS software.

Results-

Genetic alterations were found in 181 genes. APC mutations were found in $50 \%$ of the esophageal adenocarcinomas, $5 \%$ of the gastric adenocarcinomas and $33.3 \%$ of the small intestinal adenocarcinomas $(p=0.04)$. PIK3 gene family mutations were found in $10 \%$ of the gastric adenocarcinomas, $66 \%$ of the gall bladder adenocarcinomas and $66 \%$ of the small intestinal adenocarcinomas $(p=0.002)$. The mutations were found exclusively in the PIK3 class 1 family.

Conclusion-

In this study, APC gene mutations were found to be more frequent in esophageal and small intestinal adenocarcinomas than previously reported. PIK3 class 1 gene family mutations were found to be more frequent in gallbladder and small intestinal adenocarcinomas.

\section{Introduction}

The gastrointestinal tract is home to a wide variety of vastly different, diagnostically challenging neoplasms. ${ }^{1}$ When taken as a whole, adenocarcinomas of the gastrointestinal tract are one of the most common malignancies in the world. ${ }^{2}$ Though they share morphological similarities, these gastrointestinal adenocarcinomas display distinct clinical characteristics in terms of different risk factors and discrete prognostic patterns. ${ }^{3-5}$

Owing to their morphological heterogeneity, the adenocarcinomas of the gastrointestinal tract are difficult to profile based on location. ${ }^{5,6}$ Over the past two decades, immunohistochemistry has evolved and has been routinely incorporated into the workup and assessment of adenocarcinomas of the gastrointestinal 
tract. ${ }^{5}$ Attempts have been made to utilize immunohistochemical profiles for determining various tumor characteristics including the origin of a malignancy in the setting of widely metastatic disease. ${ }^{5,6}$

Multiple studies such as those summarized in a review article by Wong et al emphasize the extremely high specificity of the CK7-/CK20 + immunoprofile for colorectal cancers that effectively separates them from other gastrointestinal adenocarcinomas. ${ }^{7}$ However, the upper gastrointestinal adenocarcinomas including esophageal, gastroesophageal, gastric, small intestinal and gallbladder adenocarcinomas predominantly express CK7 and not CK20. ${ }^{7}$ Other commonly used markers such as CDX2, Villin, CK17 and MUCs exhibit heterogenous staining patterns and do not aid in differentiating between these neoplasms. ${ }^{5,8-10}$

With the advent of molecular techniques and next generation sequencing, attempts are being made to delineate distinct molecular characteristics of these morphologically related adenocarcinomas from

adjoining anatomical sites. ${ }^{4,11-14}$ Despite these efforts the molecular biology of upper gastrointestinal adenocarcinoma, still requires further elucidation. Identifying and understanding the distinct molecular pathways holds the key to finding novel diagnostic and therapeutic targets for these tumors which usually present at advanced stages which portends a poor prognosis.

\section{Materials And Methods}

This study was reviewed and approved by the Institutional Review Board and Ethics Committee of Mount Sinai Medical Center.

The aim of the present study was to investigate the clinical role and significance of a panel of genetic alterations found in adenocarcinoma cases retrieved from our pathology records.

\section{Selection of tumors for tissue profiling}

Only adenocarcinomas in which the primary site of the tumor could be established by clinical and radiological findings, were included in this study. Cases with history of another malignancy or concomitant second primary were excluded from the study.

\section{Genetic testing and data analysis}

Formalin fixed paraffin embedded tissue blocks of the tumor were selected and sent for next generation sequencing which was performed at an outside institution. The data collected from these sequencing studies included the total number and type of genetic alterations including base substitutions, copy number variations, indels and rearrangements. The obtained sequencing data was analyzed for recurrent genetic abnormalities.

After the statistically significant genetic abnormalities were detected for the adenocarcinoma cases, the data was stratified by primary sites of tumor location mentioned above. In the stratified data, each 
primary site of tumor location (each category) showed the presence or absence of pathological diagnosis for that specific site of tumor location. This was necessary to make binary logistic regression models for each primary site of tumor location.

Five binary logistic regression models were done for the five primary sites of tumor locations included in the study. This was done in order to screen for a possible association between the significant genetic abnormalities (PIK3 and APC) and the specific primary sites of tumor locations. The logistic regression models used the presence or absence of statistically significant genetic alterations as the covariate and presence or absence of pathological diagnosis (in the different primary sites of tumor locations) as the dependent variable.

\section{Statistical analysis.}

Statistical analyses were performed using IBM SPSS25 and SAS software. The study included only nonparametric data which was compared using Fisher Exact and chi square test for statistical significance. A $p$-value less than 0.05 was considered statistically significant.

\section{Results}

Thirty-seven cases of adenocarcinoma were included in this study. Of these, six were of esophageal origin, two of gastroesophageal junction origin, twenty of gastric origin, six of small intestinal origin and three were primaries from the gallbladder. The study sample consisted of 11 female patients between the ages of 40 to 87 years old and 26 male patients between the ages of 34 to 96 years old. The mean (66.2) and SD (14.6) of the age distribution lead to the normal range 37-95.4 years.

There was no significant difference between the age or sex distribution among the various groups (Table 1. Characteristics of study cases). Genetic alterations including base substitutions, insertions, deletions, copy number alterations and rearrangements were found in a total of 181 genes in the 37 cases studied (Table 2. List of genes found to be altered in all the cases of adenocarcinoma included in the study). 
Table 1

Characteristics of study cases.

\begin{tabular}{|llllll|}
\hline Characteristic & Esophagus & GE junction & Stomach & Gallbladder & Small Intestine \\
\hline Age (Mean & 72.3 & 61 & 66.7 & 64.6 & 61.3 \\
Gender (M:F) & $6: 0$ & $2: 0$ & $14: 6$ & $1: 2$ & $3: 3$ \\
APC mutation & 3 & 0 & 1 & 0 & 2 \\
TP53 mutation & 3 & 1 & 9 & 2 & 3 \\
PIK3mutation & 0 & 0 & 2 & 2 & 4 \\
Sample size & 6 & 2 & 20 & 3 & 6 \\
\hline *GE junction = Gastroesophageal junction & & & \\
\hline
\end{tabular}


Table 2

List of genes found to be altered in all the cases of adenocarcinoma included in the study.

\begin{tabular}{|c|c|c|c|c|c|c|}
\hline ABL1 & CDK1 & FAT1 & IDH2 & MSH3 & PPP2R1A & TNFAIP3 \\
\hline ABL2 & CDK2 & FBXW7 & INHBA & MSH6 & PRD1 & TOP2A1 \\
\hline AKT2 & CDK8 & FGF3 & INPP46 & MTOR & PRDC & TP53 \\
\hline APC & CDKN2A & FGF4 & IRF4 & MYCL1 & PREX2 & TSC1 \\
\hline AR & CDKN2B & FGF10 & IRS2 & MYS3 & PRKAR1A & TSC2 \\
\hline ARAF & CDX1 & FGF19 & JAK1 & NF1 & PRKC1 & U2AF1 \\
\hline ARID1A & CDX2 & FGFR1 & JAK2 & NKX2 & PRKCDC & VEGFA \\
\hline ARID1B & CEBPA & FGFR2 & JAK3 & NITCH1 & PTCH1 & VHL \\
\hline ARID2 & CHD1 & FGFR3 & KDM5A & NOTCH2 & PTEN & WISP3 \\
\hline ASXL1 & CHD2 & FGFR4 & KDR & NOTCH3 & PTPN11 & WT1 \\
\hline ATM & CHD4 & FLT1 & KEAP1 & NRAS & QK1 & XP01 \\
\hline ATR & CHEK2 & FLT3 & KIT & NTRK3 & RANBP2 & ZBTB \\
\hline ATRX & CKIT & FLT4 & KRAS & NTSC2 & RICTOR & ZNF217 \\
\hline AXIN1 & CREBBP & FOXP1 & L194R & PANCA & RMB10 & ZNF703 \\
\hline BAP1 & CSF1R & FRS2 & LRP1B & PARK2 & RNF43 & TNFAIP3 \\
\hline BCOR & CTCF & GATA3 & MAG12 & PAX5 & ROS1 & TOP2A1 \\
\hline BCORL1 & CTNNB1 & GATA4 & MAPKЗKI & PBRM1 & SDHD & TP53 \\
\hline BLM & CYLD & GATA6 & MDM2 & PDGFRA & SF3B1 & TSC1 \\
\hline BRAF & DDR2 & GF2 & MDM4 & PDGFRB & SMAD2 & TSC2 \\
\hline BRCA2 & EGFR & GLI1 & MED12 & PIK3CA & SMAD3 & \\
\hline BRD4 & EPHA3 & GNAS & MEF2B & PIK3CB & SMAD4 & \\
\hline BTK & ERBB2 & GPR124 & MLH1 & PIK3CG & SMARCA4 & \\
\hline CARD2 & ERBB3 & GRM3 & MLL1 & PIK3R1 & SNCAIP & \\
\hline CARD11 & ESR1 & HNF1A & MLL2 & PIK3R2 & Sox9 & \\
\hline CBL & EWSR1 & HSD3B1 & MLL3 & PLCG2 & SPEN & \\
\hline CCNE1 & FANCC & HSP90AA1 & MPL & POLD1 & SPTA1 & \\
\hline CD70 & FANCD2 & IDH1 & $\mathrm{MSH} 2$ & POLE & SRC & \\
\hline
\end{tabular}


APC mutations were found in $15.7 \%$ of the total adenocarcinoma cases included in the study. APC mutations were found $50 \%$ of the esophageal adenocarcinomas, $5 \%$ of the gastric adenocarcinomas and $33.3 \%$ of the small intestinal adenocarcinomas. None of the gastroesophageal junction adenocarcinomas or gallbladder adenocarcinomas had APC mutations. This difference in the frequency of APC mutations in adenocarcinomas was significant with a p-value of 0.04 . The mutations were not limited to any particular site of the APC gene.

Of the 37 adenocarcinomas, $48.6 \%$ had TP53 mutations. TP53 mutations were found in $66.7 \%$ adenocarcinomas of gallbladder, $50 \%$ adenocarcinomas of esophagus and gastroesophageal junction, $40 \%$ gastric adenocarcinomas and $33.3 \%$ small intestinal adenocarcinomas. There was no significant difference in the TP53 mutational frequency between the different locations. Only two of the tumors (one gastric and one small intestinal) had concurrent APC and TP53 mutations. The remainder of the TP53 mutant adenocarcinomas were APC wild type. $(p=0.2)$

PIK3 gene family mutations were found in $22.2 \%$ of the total adenocarcinoma cases included in the study. PIK3 gene family mutations were found in $10 \%$ of the gastric adenocarcinoma, $66 \%$ of the gallbladder adenocarcinomas and $66 \%$ of the small intestinal adenocarcinomas. This difference in the frequency of PIK3 mutations in adenocarcinomas was significant with a p-value of 0.002 . The mutations were found exclusively in PIK3 class 1 family in all cases; PIK3CA was mutated in two small intestinal and one gastric adenocarcinoma, PIK3CB was mutated in one small intestinal and one gastric adenocarcionoma, PIK3CG was mutated in one small intestinal adenocarcinoma and PIK3R1/R2 in the two gallbladder adenocarcinomas. Of the 8 PIK3 mutant tumors in this study, only two (both small intestinal) adenocarcinomas had concomitant APC mutations. The remainder PIK3 mutant tumors were APC wild type.

The logistic regression model for the small intestine primary tumor location was the only model that satisfied the convergence criterium and that was statistically significant with a concordance-statistic value of 0.76 (Table 3. Association of predicted probabilities and observed responses for the small intestine site of tumor location model). The Wald Chi-Square test, the Likelihood Ratio Chi-Square test and the Score Chi-Square Test were all statistically significant with $p$-values of $0.03,0.02$ and 0.011 respectively (Table 4. Testing global null hypothesis for association between APC and PIK3 genetic abnormalities and small intestine site of tumor location). The model also shows the analysis of maximum likelihood estimates which was significant for the PIK3 gene family mutation with a p-value of 0.01 but not for the APC mutation (Table 5. Analysis of maximum likelihood estimates for the small intestine site of tumor location model). Also, statistically significant values were found for the PIK3 mutation in the $95 \%$ Wald confidence interval (Cl): 1.63-96.2, but not for the APC mutation (Table 6. Odds ratio estimates for the small intestine site of tumor location model). 
Table 3

Association of predicted probabilities and observed responses for the small intestine site of tumor location model.

\begin{tabular}{|llll|}
\hline Percent Concordant & $\mathbf{6 2 . 2}$ & Somers' D & $\mathbf{0 . 5 3 3}$ \\
\hline Percent Discordant & 8.9 & Gamma & 0.750 \\
\hline Percent tied & 28.9 & Tau-a & 0.152 \\
\hline Pairs & 180 & C & 0.767 \\
\hline
\end{tabular}

Table 4

Testing global null hypothesis for association between APC and PIK3 genetic abnormalities and small intestine site of tumor location (Beta $=0)$.

\begin{tabular}{|llll|}
\hline Test & Chi-Square & DF & Pr $>$ ChiSq \\
\hline Likelihood Ratio & 7.7306 & 2 & 0.0210 \\
\hline Score & 8.9739 & 2 & 0.0113 \\
\hline Wald & 6.4754 & 2 & 0.0393 \\
\hline
\end{tabular}

Table 5

Analysis of maximum likelihood estimates for the small intestine site of tumor location model.

\begin{tabular}{|llllll|}
\hline Parameter & DF & Estimate & Standard error & Wald Chi-Square & Pr $>$ ChiSq \\
\hline Intercept & 1 & -2.7906 & 0.8140 & 11.7535 & 0.0006 \\
APC & 1 & 1.0792 & 1.1944 & 0.8164 & 0.3662 \\
\hline PIK3 & 1 & 2.5304 & 1.0389 & 5.9329 & 0.0149 \\
\hline
\end{tabular}

Table 6

Odds ratio estimates for the small intestine site of tumor location model.

\begin{tabular}{|lllc|}
\hline Effect & Point estimate & \multicolumn{2}{c|}{$\mathbf{9 5 \%}$ Wald Confidence limits } \\
\hline APC & 2.942 & 0.283 & 30.576 \\
\hline PIK3 & 12.559 & 1.639 & 96.209 \\
\hline
\end{tabular}

\section{Discussion}

The gastrointestinal tract includes the luminal organs extending from the esophagus to the rectum in addition to the pancreas and the gall bladder.15 In addition to the sizeable cellular mass, the epithelium has a rapid turnover, laying the foundation for gastrointestinal cancers that are among the most frequent malignancies resulting in mortality. 16 These tumors usually present late with metastatic disease. Though 
morphologically similar, adenocarcinomas of different sites in the gastrointestinal tract are clinically distinct with unique risk factors and dissimilar prognostic behavior.3-5

The determination of the primary site of these adenocarcinomas within the luminal GI tract in the setting of widely metastatic disease has remained challenging despite the advances in immunohistochemistry. ${ }^{5,6}$ The CK7-/CK20 + immune-profile has good specificity for differentiating colorectal adenocarcinoma from other gastrointestinal adenocarcinomas. ${ }^{7,17-18}$ Similarly, pancreatic ductal adenocarcinomas display a CK7+/CK20 + immune-profile along with substantially higher CK17 expression. ${ }^{19-20}$

But when it comes to the upper gastrointestinal adenocarcinomas including those of the esophagus, gastroesophageal junction, stomach, small intestine and gallbladder, immunohistochemical profiles are indistinct and overlapping. CK7 is usually positive in most of them while CK 20 is usually negative. ${ }^{7}$ Other markers such as CDX2, Villin, CK17 and MUCs do not display specific staining patterns and aren't helpful in differentiating these adenocarcinomas when used individually or as a panel. ${ }^{5,8-10,21}$

The emergence of high throughput technologies such as next generation sequencing technology has heralded the beginning of the genomic era. Novel genomic and epigenomic biomarkers and signatures are being discovered and developed for early detection and prognosis of gastrointestinal cancers. ${ }^{22}$ In addition, scientists are endeavoring to understand how different molecular events lead to varying biologic properties and clinical features of these cancers based on different cells and tissues of origin. ${ }^{23}$

Morphologically similar tumors with similar immunohistochemical profiles arising in different organs may be from identical preceding events driving the tumorigenesis. However, even morphologically identical tumors arising in different organs differ substantially not only in terms of the oncogenic threats and environmental risk factors but also in cellular dynamics and tumorigenic potential. These inter-tumor dissimilarities, when identified, can not only improve diagnostic accuracy but also identify therapeutic targets and further patient welfare using precision medicine. ${ }^{23}$

A comprehensive comparative analysis of genetic alterations identified by high throughput sequencing can potentially uncover tissue specific determinants in different gastrointestinal adenocarcinomas which can translate to differences in prognosis and may help direct therapeutic decisions. Presence or absence of certain gene mutations and/or varying mutational frequency result in tissue specific mutational signatures. ${ }^{23}$

Beta-catenin, a protein coded by the CTNNB1 gene, is integral in intercellular adhesion and signal transduction and its degradation is regulated largely by adenomatous polyposis coli (APC) gene. Mutation in either of these can cause aberrant accumulation of beta catenin leading to increased transcription of downstream target proteins of the wingless integration site family member (WNT) signaling pathway such as MYC and CCND1. ${ }^{24}$ The dysregulation of the APC/beta-catenin and WNT signaling pathway is an integral mechanism of tumorigenesis in several cancers, most prominently in 
colorectal carcinomas. ${ }^{25-27}$ Choi et al reported a very low frequency of APC/beta catenin mutations in their study analyzing esophageal and esophagogastric junction adenocarcinomas based on partial screening mutational analyses. ${ }^{27}$

In our study, APC gene mutations were found to be most frequent in esophageal adenocarcinomas followed by small intestinal adenocarcinomas and infrequent in gastric adenocarcinomas. Choi et al reported similar findings in esophageal adenocarcinomas in their study in 97 tumors. ${ }^{27}$ Salem et al reported similarly low frequency of APC mutations in gastric adenocarcinomas but found significantly lower frequency of APC mutations in esophageal adenocarcinomas. ${ }^{14}$ The reported frequency of APC mutations in gastric adenocarcinomas varies widely. Fang et al reported an APC mutation frequency of $25 \%$ in gastric adenocarcionmas. ${ }^{28}$ Rokutan et al in their study of 43 gastric intramucosal adenocarcinomas found a higher frequency of APC mutations than in our study. ${ }^{29}$ However, they reported that the APC and TP53 mutations were mutually exclusive. This is reflected in our study where $88.9 \%$ of the TP53 mutated adenocarcinomas were APC wild type though this finding did not reach statistical significance. This might be attributed to the low number of study subjects and might reach statistical significance if a higher number of cases were evaluated supporting the existence of TP53/APC molecular subsets. Similar to gastric adenocarcinomas, wide variations have been reported in APC gene mutations in small intestinal adenocarcinomas. Schrock et al found the APC mutational frequency to be around $26 \%$ in 317 small intestinal adenocarcinomas studied. ${ }^{30}$ Similar findings were reported by Hanninen et al. ${ }^{31}$ However, in a recent study, Ota et al reported a much higher frequency of APC mutations in small intestinal adenocarcinomas. ${ }^{32}$ Our findings are comparable to the APC mutational frequency reported by Ota et al. The difference in APC mutational frequency among different studies may be ascribed to locational differences. The reported incidence of APC mutation in duodenal adenocarcinomas is much lower as compared to other small intestinal locations. ${ }^{32}$ The majority of adenocarcinomas in the study by Schrock et al were duodenal. ${ }^{30}$ However, this cannot be ascertained as the small intestinal adenocarcinomas were not further stratified based on location in our study due to the small number of small intestinal adenocarcinomas included. None of the gastroesophageal junction adenocarcinomas or gallbladder adenocarcinomas included in this study had any mutations in the APC gene and this low incidence is similar to those reported previously. ${ }^{27,33}$

PIK3 gene mutations were found to be relatively more frequent in small intestinal and gall bladder adenocarcinomas as compared to esophageal and gastric adenocarcinomas. All the PIK3 gene family mutations were limited to class 1 PIK3 genes with the majority localizing to PIK3CA as reported in previous studies. ${ }^{34-38}$ PIK3CA mutations are reported to occur in $8-10 \%$ cancers. ${ }^{39}$ Disturbances in the PIK3 signaling pathway and its regulation are known to underlie numerous human diseases. Activating mutations in the genes encoding the catalytic subunits of class IA PIK3 have been reported in several cancer types. ${ }^{40}$ The frequency of PIK3 mutations in esophageal and gastric adenocarcinomas has been reported to be low in multiple studies as reflected in our study. ${ }^{14,34-35}$ 
The previously reported frequency of PIK3 gene family mutations in gallbladder adenocarcinoma is much lower than found in our study. ${ }^{36,37}$ Though, this could represent a sampling bias due to the small number of gallbladder adenocarcinoma cases included in this study, a more plausible explanation for the higher than reported frequency of PIK3 gene family mutations is the inclusion of mutations in all the class 1 genes. The mutations in the PIK3 gene family in gallbladder adenocarcinomas were limited to the class 1 regulatory subunit 1 and 2 genes. All class IA catalytic subunits interact and are controlled by regulatory subunits, and mutations/deletions in these regulatory subunits have been identified in multiple cancers. ${ }^{40}$ Though the role of PIK3R1 and PIK3R2 mutations in gallbladder adenocarcinoma has so far not been described, they are known oncogenic drivers in endometrial adenocarcinoma where gain of function mutations in PIK3R2 results in oncogenesis via PTEN stabilization. ${ }^{41,42}$

Again, like for gallbladder adenocarcinoma, the frequency of PIK3 family mutations in small intestinal adenocarcinoma was found to be much higher in our study when compared to previously published data. ${ }^{30-31,38}$ The majority of these mutations were in PIK3CA as previously reported however mutations were also found in PIK3CG and PIK3CB genes accounting for the higher than reported incidence. Though the incidence of PIK3CA mutation in intestinal adenocarcinomas is reportedly low, the PI3K/AKT pathway is the most mutated pathway, where at least one gene was mutated in the majority of small intestinal adenocarcinomas. ${ }^{31}$ Hare et al in their study showed that the most common PIK3CA mutation (Pik3caH1047R seen in colorectal carcinomas), when expressed at physiological levels, is insufficient to initiate intestinal tumorigenesis. However, when acting in tandem with APC loss, it promotes the development of invasive adenocarcinomas in the small intestine. ${ }^{43}$ This tandem effect is seen on the logistic regression model in small intestinal adenocarcinomas in our study, though the statistical significance is limited by the small study set.

In addition to the small study population that remains a limitation of this study, the association of different mutations with the histo-morphological types of adenocarcinomas was not assessed. Also, the association between the various mutations and presence or absence of precusor lesions was not assessed.

\section{Conclusions}

In this study on luminal upper gastrointestinal adenocarcinomas, APC gene mutations were found to be more frequent in esophageal and small intestinal adenocarcinomas than previously reported. PIK3 class 1 gene family mutations, when taken as a group, were found to be more frequent in gallbladder and small intestinal adenocarcinomas. Though a majority of the cases had mutations in PIK3CA, mutations in other genes of the PIK3 class 1 family, namely PIK3CB, PIK3CG, PIK3R1 and PIK3R2 were also identified in a subset of cases.

\section{Declarations}

1. Funding- Nil. 
2. Conflicts of interest/ competing interest-The authors declare they have no conflicts of interest.

3. Ethics approval-This study was approved by the IRB at MSMC, FL (FWA00000176).

4. Consent to participate- Not applicable (retrospective data analysis)

5. Consent to publish-Not applicable (retrospective data analysis)

6. Availability of data and material- Not applicable.

7. Code availability- Not applicable.

8. Author contributions- All authors contributed to the study conception and design. Material preparation, data collection and analysis were performed by Kritika Krishnamurthy and Sophia Navajas Urioste. The first draft of the manuscript was written by Kritika Krishnamurthy and all authors commented on previous versions of the manuscript. All authors read and approved the final manuscript.

\section{References}

1. Bray F, Ferlay J, Soerjomataram I, Siegel RL, Torre LA, Jemal A. Global cancer statistics 2018 : GLOBOCAN estimates of incidence and mortality worldwide for 36 cancers in 185 countries. CA Cancer J Clin 2018,68(6):394-424.

2. Fitzmaurice $C$, Dicker D, Pain A, Hamavid H, Moradi-Lakeh M, Maclntyre MF, et al. The Global Burden of Cancer 2013. JAMA Oncol. 2015 Jul,1(4):505-27.

3. Weinberg RA. The nature of cancer. Zayatz E, editor. The biology of cancer, 2 nd ed. Garland Science. Taylor \& Francis Group, LLC, 2014. p. 34-6.

4. Lin J, Beerm DG. Molecular biology of upper gastrointestinal malignancies. Semin Oncol. 2004 Aug,31(4):476-86.

5. Wong HH, Chu P. Immunohistochemical features of the gastrointestinal tract tumors. J Gastrointest Oncol. 2012 Sep,3(3):262-84.

6. Park SY, Kim HS, Hong EK, et al. Expression of cytokeratin 7 and 20 in arcinomas of the stomach and colorectum and their value in the differential diagnosis of metastatic carcinomas to the ovary. Hum Pathol. 2002,33:1078-1085.

7. Tot T. Identifying colorectal metastases in liver biopsies: the novel CDX2 antibody is less specific than the cytokeratin 20_/7- phenotype. Med Sci Monit. 2004,10:BR139-BR143

8. Kende Al, Carr NJ, Sobin LH. Expression of cytokeratins 7 and 20 in carcinomas of the gastrointestinal tract. Histopathology. 2003,42:1137-1140.

9. Flucke U, Steinborn E, Dries V, et al. Immunoreactivity of cytokeratins (CK7, CK20) and mucin peptide core antigens (MUC1, MUC2, MUC5AC) in adenocarcinomas, normal and metaplastic tissues of the distal oesophagus, oesophago-gastric junction and proximal stomach. Histopathology 2003,43:12734. 
10. Goldstein NS, Long A, Kuan SF, et al. Colon signet ring cell adenocarcinoma: immunohistochemical characterization and comparison with gastric and typical colon adenocarcinomas. Appl Immunohistochem Mol Morphol 2000,8:183-8.

11. D'Errico M, Alwers E, Zhang Y, Edelmann D, Brenner H, Hoffmeister M. Identification of prognostic DNA methylation biomarkers in patients with gastrointestinal adenocarcinomas: A systematic review of epigenome-wide studies. Cancer Treat Rev. 2020 Jan,82:101933.

12. Schrock AB, Devoe CE, McWilliams R, Sun J, Aparicio T, Stephens PJ, Ross JS, Wilson R, Miller VA, Ali SM, Overman MJ. Genomic Profiling of Small-Bowel Adenocarcinoma. JAMA Oncol. 2017 Nov 1,3(11):1546-1553.

13. Tokunaga R, Xiu J, Johnston C, Goldberg RM, Philip PA, Seeber A, Naseem M, Lo JH, Arai H, Battaglin F, Puccini A, Berger MD, Soni S, Zhang W, Hwang JJ, Shields AF, Marshall JL, Baba H, Korn WM, Lenz HJ. Molecular Profiling of Appendiceal Adenocarcinoma and Comparison with Right-sided and Leftsided Colorectal Cancer. Clin Cancer Res. 2019 May 15,25(10):3096-3103.

14. Salem ME, Puccini A, Xiu J, Raghavan D, Lenz HJ, Korn WM, Shields AF, Philip PA, Marshall JL, Goldberg RM. Comparative Molecular Analyses of Esophageal Squamous Cell Carcinoma, Esophageal Adenocarcinoma, and Gastric Adenocarcinoma. Oncologist. 2018 Nov,23(11):13191327.

15. Bijlsma MF, Sadanandam A, Tan P, Vermeulen L. Molecular subtypes in cancers of the gastrointestinal tract. Nat Rev Gastroenterol Hepatol. 2017 Jun,14(6):333-342.

16. Siegel RL, Miller KD, Jemal A. Cancer statistics, 2016. CA Cancer J. Clin. 2016, 66: 7-30.

17. Rullier A, Le Bail B, Fawaz R, et al. Cytokeratin 7 and 20 expression in cholangiocarcinomas varies along the biliary tract but still differs from that in colorectal carcinoma metastases. Am J Surg Pathol. 2000,24:870-876.

18. Park SY, Kim HS, Hong EK, et al. Expression of cytokeratin 7 and 20 in arcinomas of the stomach and colorectum and their value in the differential diagnosis of metastatic carcinomas to the ovary. Hum Pathol. 2002,33:1078-1085.

19. Sarbia M, Fritze F, Geddert H, von Weyhern C, Rosenberg R, Gellert K. Differentiation between pancreaticobiliary and upper gastrointestinal adenocarcinomas: is analysis of cytokeratin 17 expression helpful? Am J Clin Pathol. 2007 Aug,128(2):255-9.

20. Chu P, Wu E, Weiss LM. Cytokeratin 7 and cytokeratin 20 expression in epithelial neoplasms: a survey of 435 cases. Mod Pathol. 2000,13:962-972.

21. Chu PG, Chung L, Weiss LM, Lau SK. Determining the site of origin of mucinous adenocarcinoma: an immunohistochemical study of 175 cases. Am J Surg Pathol. 2011 Dec,35(12):1830-6.

22. Sharma KL, Bhatia V, Agarwal P, Kumar A. Gastrointestinal Cancers: Molecular Genetics and Biomarkers. Can J Gastroenterol Hepatol. 2018 Oct 18,2018:4513860.

23. Bijlsma MF, Sadanandam A, Tan P, Vermeulen L. Molecular subtypes in cancers of the gastrointestinal tract. Nat Rev Gastroenterol Hepatol. 2017 Jun,14(6):333-342.

24. Clevers H, Nusse R. Wnt/ $\beta$-catenin signaling and disease. Cell. 2012, 149:1192-1205. 
25. Kinzler, KW., Vogelstein, B. Colorectal tumors. In: Vogelstein, B., Kinzler, KW., editors. The genetic basis of human cancer. 2. New York: McGraw-Hill, 2002. p. 583-612.

26. Goss KH, Groden J. Biology of the adenomatous polyposis coli tumor suppressor. J Clin Oncol. 2000, 18:19671979.

27. Choi YW, Heath El, Heitmiller R, Forastiere AA, Wu TT. Mutations in beta-catenin and APC genes are uncommon in esophageal and esophagogastric junction adenocarcinomas. Mod Pathol. 2000 Oct,13(10):1055-9.

28. Fang Z, Xiong Y, Li J, Liu L, Zhang W, Zhang C, Wan J. APC gene deletions in gastric adenocarcinomas in a Chinese population: a correlation with tumour progression. Clin Transl Oncol. 2012 Jan,14(1):60-5.

29. Rokutan H, Abe H, Nakamura H, Ushiku T, Arakawa E, Hosoda F, Yachida S, Tsuji Y, Fujishiro M, Koike $\mathrm{K}$, Totoki Y, Fukayama M, Shibata T. Initial and crucial genetic events in intestinal-type gastric intramucosal neoplasia. J Pathol. 2019 Apr,247(4):494-504.

30. Schrock AB, Devoe CE, McWilliams R, Sun J, Aparicio T, Stephens PJ, Ross JS, Wilson R, Miller VA, Ali SM, Overman MJ. Genomic Profiling of Small-Bowel Adenocarcinoma. JAMA Oncol. 2017 Nov 1,3(11):1546-1553.

31. Hänninen UA, Katainen R, Tanskanen T, et al. Exome-wide somatic mutation characterization of small bowel adenocarcinoma. PLoS Genet 2018, 14: e1007200.

32. Ota R, Sawada T, Tsuyama S, Sasaki Y, Suzuki H, Kaizaki Y, Hasatani K, Yamamoto E, Nakanishi H, Inagaki S, Tsuji S, Yoshida N, Doyama H, Minato H, Nakamura K, Kasashima S, Kubota E, Kataoka H, Tokino T, Yao T, Minamoto T. Integrated genetic and epigenetic analysis of cancer-related genes in non-ampullary duodenal adenomas and intramucosal adenocarcinomas. J Pathol. 2020 Nov,252(3):330-342.

33. Rashid A, Gao YT, Bhakta S, Shen MC, Wang BS, Deng J, Fraumeni JF Jr, Hsing AW. Beta-catenin mutations in biliary tract cancers: a population-based study in China. Cancer Res. $2001 \mathrm{Apr}$ 15,61(8):3406-9.

34. Essakly A, Loeser $H$, Kraemer M, Alakus $H$, Chon SH, Zander T, Buettner R, Hillmer AM, Bruns CJ, Schroeder W, Gebauer F, Quaas A. PIK3CA and KRAS Amplification in Esophageal Adenocarcinoma and their Impact on the Inflammatory Tumor Microenvironment and Prognosis. TransI Oncol. 2020 Feb,13(2):157-164.

35. Phillips WA, Russell SE, Ciavarella ML, Choong DY, Montgomery KG, Smith K, Pearson RB, Thomas RJ, Campbell IG. Mutation analysis of PIK3CA and PIK3CB in esophageal cancer and Barrett's esophagus. Int J Cancer. 2006 May 15,118(10):2644-6.

36. Narayan RR, Creasy JM, Goldman DA, Gönen M, Kandoth C, Kundra R, Solit DB, Askan G, Klimstra DS, Basturk O, Allen PJ, Balachandran VP, D'Angelica MI, DeMatteo RP, Drebin JA, Kingham TP, Simpson AL, Abou-Alfa GK, Harding JJ, O'Reilly EM, Butte JM, Matsuyama R, Endo I, Jarnagin WR. Regional differences in gallbladder cancer pathogenesis: Insights from a multi-institutional comparison of tumor mutations. Cancer. 2019 Feb 15,125(4):575-585. 
37. Deshpande V, Nduaguba A, Zimmerman SM, Kehoe SM, Macconaill LE, Lauwers GY, Ferrone C, Bardeesy N, Zhu AX, Hezel AF. Mutational profiling reveals PIK3CA mutations in gallbladder carcinoma. BMC Cancer. 2011 Feb 8,11:60.

38. Schönleben F, Qiu W, Allendorf JD, Chabot JA, Remotti HE, Su GH. Molecular analysis of PIK3CA, BRAF, and RAS oncogenes in periampullary and ampullary adenomas and carcinomas. $J$ Gastrointest Surg. 2009 Aug,13(8):1510-6.

39. Janku F, Hong DS, Fu S, et al. Assessing PIK3CA and PTEN in early-phase trials with PI3K/AKT/mTOR inhibitors. Cell Rep. 2014,6(2):377-387.

40. Dornan GL, Burke JE. Molecular Mechanisms of Human Disease Mediated by Oncogenic and Primary Immunodeficiency Mutations in Class IA Phosphoinositide 3-Kinases. Front Immunol. 2018,9:575.

41. Vallejo-Díaz J, Chagoyen M, Olazabal-Morán M, González-García A, Carrera AC. The Opposing Roles of PIK3R1/p85a and PIK3R2/p85 $\beta$ in Cancer. Trends Cancer. 2019 Apr,5(4):233-244.

42. Cheung LW, Hennessy BT, Li J, Yu S, Myers AP, Djordjevic B, Lu Y, Stemke-Hale K, Dyer MD, Zhang F, Ju Z, Cantley LC, Scherer SE, Liang H, Lu KH, Broaddus RR, Mills GB. High frequency of PIK3R1 and PIK3R2 mutations in endometrial cancer elucidates a novel mechanism for regulation of PTEN protein stability. Cancer Discov. 2011 Jul,1(2):170-85. doi: 10.1158/2159-8290.CD-11-0039.

43. Hare LM, Phesse TJ, Waring PM, Montgomery KG, Kinross KM, Mills K, Roh V, Heath JK, Ramsay RG, Ernst M, Phillips WA. Physiological expression of the PI3K-activating mutation Pik3ca(H1047R) combines with Apc loss to promote development of invasive intestinal adenocarcinomas in mice. Biochem J. 2014 Mar 1,458(2):251-8. 